\title{
Late Oesteoradionecrosis of Mandible Five Years after Radiotherapy for Carcinoma Base of Tongue
}

\author{
Somesh Mozumder, ${ }^{1}$ Shirish Dubey, ${ }^{1}$ Aniruddha Dam, ${ }^{1}$ Anup Kumar Bhowmick ${ }^{1}$
}

\section{Introduction}

\section{$\underline{\text { ABSTRACT }}$}

Osteoradionecrosis (ORN) is a process where irradiated bone undergoes necrosis and becomes exposed through soft-tissue. It is a late effect of radiotherapy. Early presentation within 2 years, is thought to be related to high dose of radiation therapy, whereas late presentation is usually secondary to trauma and delayed wound healing with in compromised tissue.

We present a case of late presentation where there was no history of trauma to the associated region; therefore making the case unique.

\section{Case report}

50 years old male patient from rural Bengal presented with history of invasive squamous cell carcinoma (SCC) of right base tongue with bilateral cervical nodal metastasis treated with external beam radiotherapy of $70 \mathrm{~Gy}$ in 35 fractions in 2009. In April 2014 he developed pain in the right side of lower jaw with foul breath and progressive difficulty and pain while opening mouth. Biopsy established the diagnosis of oesteoradionecrosis.

Discussion

ORN can be spontaneous, but most commonly results from tissue injury. The absence of reserve reparative capacity is a result of the prior radiation injury. The irradiated mandible, periosteum, and overlying soft tissue undergo hyperemia, inflammation, and endarteritis.

\section{Conclusion}

During follow up any lesion suspicious of recurrence or second malignancy with clinical features of pain, swelling, trismus, halitosis, mucosal ulceration, bare bone etc a differential diagnosis of oesteoradionecrosis should be kept in mind, even long time after radiotherapy \& absence of trauma.

$\underline{\text { Kevwords }}$

Mandible; Osteoradionecrosis; Radiation Injuries

$\mathrm{O}$ steoradionecrosis (ORN) is a process where irradiated bone undergoes necrosis and becomes exposed through soft-tissue. The first report of ORN of the jaws after radiation therapy (RT) was published in 1992 by Regaurd. ${ }^{1}$ Ewing in 1926 first recognized and reported the bone changes associated with RT and described this disease state as radiation osteitis.

ORN is a late effect of radiotherapy. ${ }^{2}$ Early presentation within 2 years, is thought to be related to high dose of Radiation therapy ( $>60$ gray), whereas late presentation is usually secondary to trauma and delayed wound healing with in compromised tissue. ${ }^{3}$

We present a case of late presentation where there was no history of trauma to the associated region.

\section{Case report}

A 50 years old male patient from rural Bengal presented with history of invasive squamous cell carcinoma (SCC) of right side of base of tongue with bilateral cervical nodal metastasis, for which he received external beam radiotherapy (EBRT) of $70 \mathrm{~Gy}$ in 35 fractions with Cobalt 60 machine in 2009, including oropharynx and both side of neck. No chemotherapy was added to the radiation.

1 - Department of ENT, Chittaranjan National Cancer
Institute, Kolkata
Corresponding author:
$\begin{aligned} & \text { Dr Somesh Mozumder } \\ & \text { email: drsomeshmozumder@gmail.com }\end{aligned}$

1 - Department of ENT, Chittaranjan National Cancer

\section{Corresponding author:}

email: drsomeshmozumder@gmail.com 


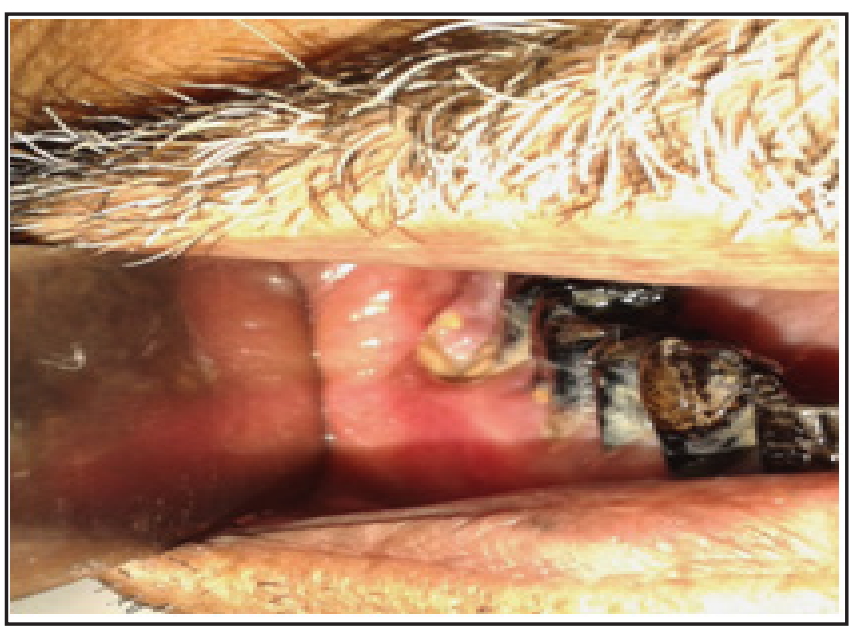

Fig.1. Mucosal oedema and ulceration with visualisation of bare bone.

The retromolar region of mandible was included in the radiation field.

No Chemotherapy was administered either before or after RT.

Since then the patient was under close follow up as per follow up schedule for head-neck cancers.

In April 2014 he developed pain in the right side of lower jaw with foul breath and progressive difficulty and pain while opening mouth.

On examination the soft tissue and skin over the right side of body of mandible near attachment of masseter muscle was found to be thickened and tender. Trismus and halitosis were noted. Oral cavity examination revealed mucosal oedema and ulceration with visualisation of bare bone through it covered by slough, (but no frank growth) over the outer part of right side of body of mandible at gingivo-buccal sulcus near lower right 2 nd and 3rd molar teeth.

There was no loco-regional lesion clinically suspicious of malignancy seen in oral cavity, oropharynx and larynx; no neck node was also palpable.

Punch biopsy was taken from the said ulceration but came inconclusive.

C.T scan and Orthopantomogram of oral cavity was taken and they both showed radiolucent area of bone loss around the said region but absence of any adjacent soft tissue mass lesion that has caused mandibular bone destruction or cortical expansion.

Re-biopsy was taken from the ulcerated area, this time with piece of exposed bone in the specimen. It showed presence of necrotic bone along with presence of inflammatory cells and granulation tissue in adjacent soft tissue.

Clinical, radiological and histological picture established the diagnosis of late oesteoradionecrosis.

Scope of using hyperbaric oxygen was not available in the institute therefore sequestrectomy was done and the patient was subjected to antibiotics, zolindronate

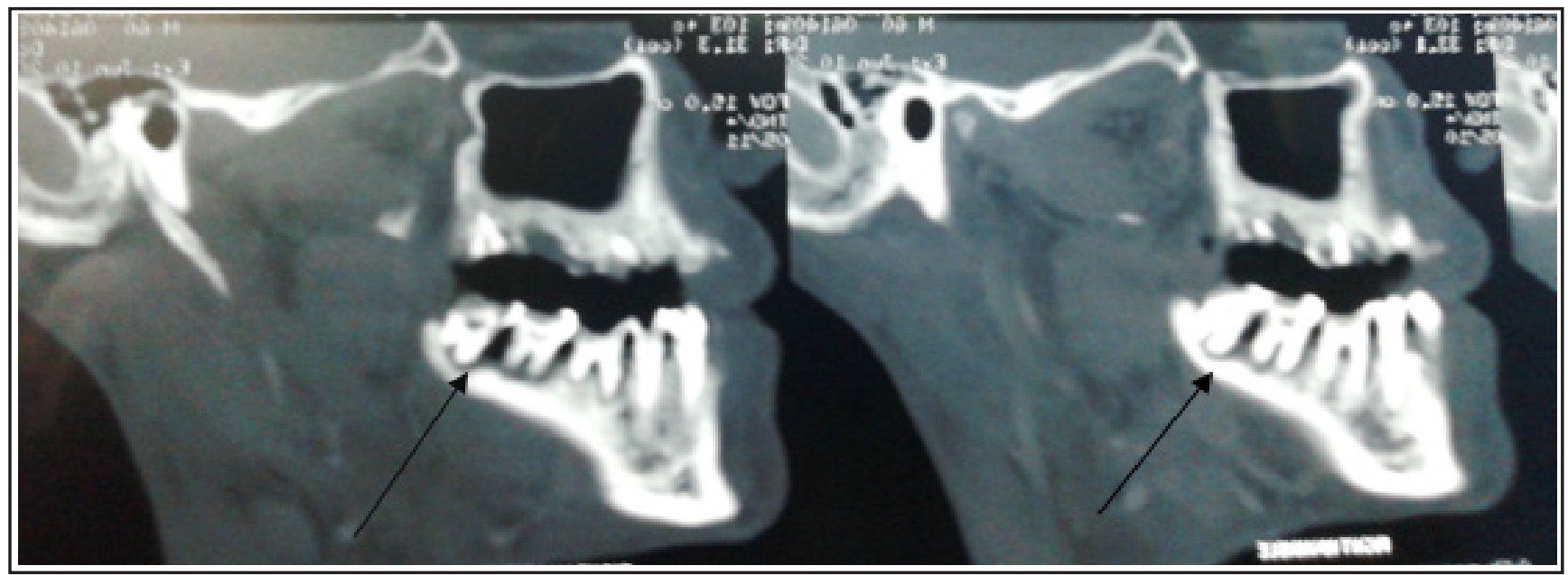

Fig.2. C.T scan of oral cavity showed radiolucent area of bone loss around lower right 2 nd and 3 rd molar teeth but absence of any adjacent soft tissue mass lesion that has caused mandibular bone destruction or cortical expansion. 


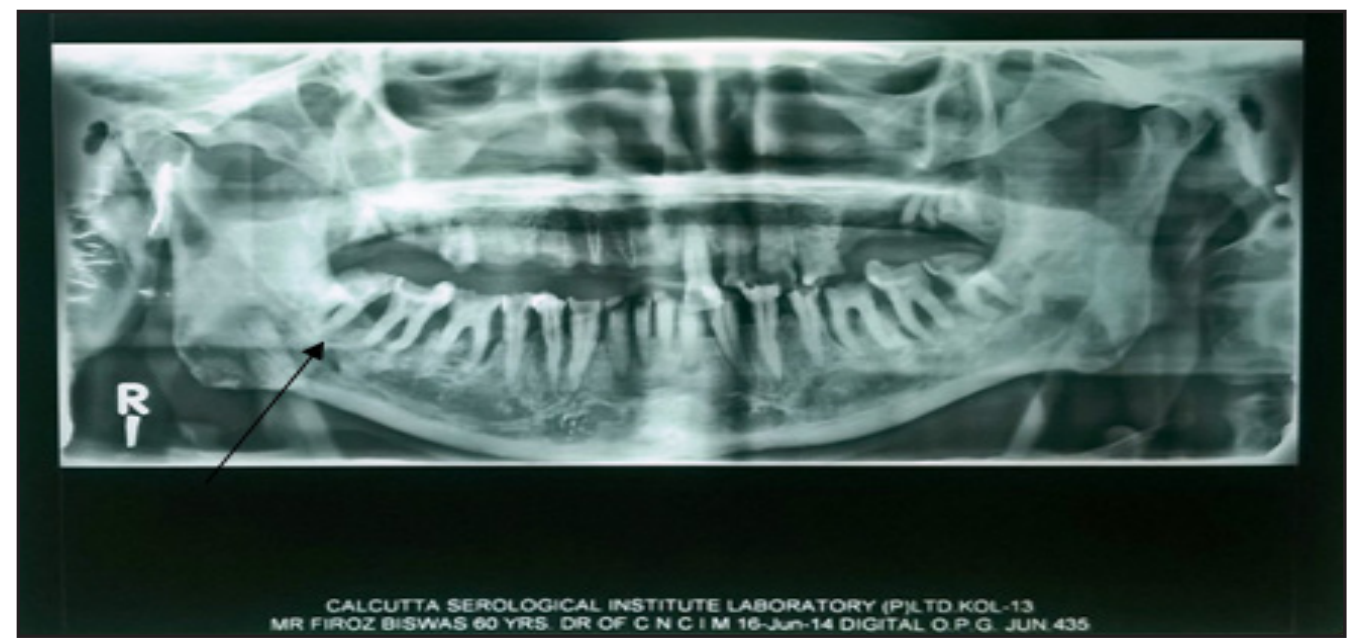

Fig.3. Orthopantomogram showing radiolucent area of bone loss around lower right 2 nd and 3rd molar teeth but absence of any adjacent soft tissue mass lesion that has caused mandibular bone destruction or cortical expansion.

and pentoxifyline and kept under close follow up.

\section{Discussion}

Osteoradionecrosis (ORN) is a condition of dead bone in a site of radiation injury. ORN can be spontaneous, but most commonly results from tissue injury. The absence of reserve reparative capacity is a result of the prior radiation injury. Even apparently trivial trauma such as denture-related injury, ulcers, or tooth extraction can overwhelm the reparative capacity of the radiationinjured bone.

Traditionally, 3 grades of disease (I, II, III) are recognized. Grade I ORN is the most common presentation. Exposed alveolar bone is observed. Grade II is that does not respond to hyperbaric oxygen (HBO) therapy and requires sequestrectomy/saucerization. Grade III is demonstrated by full-thickness involvement and/or pathologic fracture. ${ }^{1}$

The mandible is affected more often than the maxilla or any other bones of head and neck region. The incidence of ORN in the mandible is reported to be between $2 \%$ and $22 \%$ and most often affects the body of the mandible. ${ }^{1}$ The irradiated mandible, periosteum, and overlying soft tissue undergo hyperemia, inflammation, and endarteritis. ${ }^{4}$ These conditions ultimately lead to thrombosis, cellular death, progressive hypovascularity, and fibrosis. The radiated bed is hypocellular and devoid of fibroblasts, osteoblasts, and undifferentiated osteocompetent cells.

Mandibular ORN develops most commonly after local trauma, such as dental extractions, biopsies, related cancer surgery, and periodontal procedures, but it may also occur spontaneously.

Clinical symptoms include the following: pain, swelling, trismus, exposed bone, pathologic fracture, malocclusion, oral cutaneous fistula formation.

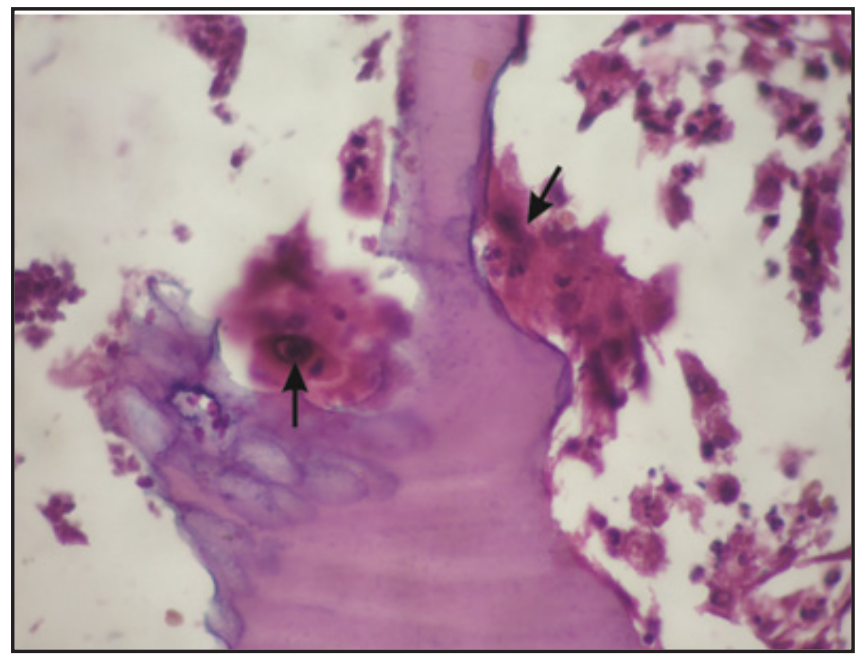

Fig.4: Histopathology showed presence of necrotic bone along with presence of inflammatory cells and granulation tissue in adjacent soft tissue. 
On physical examination, missing hair follicles, surface texture changes, and colour changes are common findings that assist clinicians in assessment of the area of radiation injury.

Treatment conventionally consists of various conservative measures, including use of long-term antibiotics, zolindronate, pentoxyfiline, local wound irrigation, debridement, sequestrectomy and hyperbaric oxygen therapy. ${ }^{2,5}$

3D conformational radiation therapy and intensity modulated radio therapy can maximize delivery to the affected area and minimize dose to the surrounding normal tissue. All patients should undergo prophylactic oral care prior to, during and completion of RT. During early post-treatment period, patient should visit the dentist every 4 months. ${ }^{3,5}$

\section{Conclusion}

At the end we want to conclude that in post RT case of CA oral cavity, during long term follow up even after five years or more any lesion suspicious of recurrence or 2nd malignancy with clinical features of pain, swelling, trismus, halitosis, mucosal ulceration, bare bone etc but with a biopsy inconclusieve of malignancy ; even in absence of trauma a differential diagnosis of oesteoradionecrosis should be kept in mind \&should be investigated accordingly.

\section{References}

1. Lyons A, Ghazali N. Osteoradionecrosis of the jaws: Current understanding of its pathophysiology and treatment. Br J Oral Maxillofac Surg, 2008;46:653-60

2. Kahenasa N, Sung EC, Nabili V, Kelly J, Garrett N, Nishimura I. Resolution of pain and complete healing of mandibular osteoradionecrosis using pentoxifylline and tocopherol: A case report. Oral Surg Oral Med Oral Pathol Oral Radiol, 2012;113:e18-23

3. Rayatt SS, Mureau MA, Hofer SO. Osteoradionecrosis of mandible: Etiology, prevention, diagnosis and treatment. Indian J Plast Surg. 2007; 40:s65-71

4. Bras J, de Jonge HK, van Merkesteyn JP. Osteoradionecrosis of the mandible: Pathogenesis. Am J Otolaryngol. 1990; 11:24450

5. Jacobson AS, Buchbinder D, Hu K, Urken ML. Paradigm shifts in the management of osteoradionecrosis of the mandible. Oral Oncol, 2010;46:795-801. 